\title{
2,3,8,12,13-Pentamethoxy-5H-dibenzo[c,n]acridin-7(6H)-one toluene solvate
}

\author{
Marlon R. Lutz Jr \\ Loyola University Chicago \\ Matthias Zeller \\ Youngstown State University \\ Daniel P. Becker Ph.D. \\ Loyola University Chicago, dbecke3@luc.edu
}

Follow this and additional works at: https://ecommons.luc.edu/chemistry_facpubs

Part of the Chemistry Commons

\section{Author Manuscript}

This is a pre-publication author manuscript of the final, published article.

\section{Recommended Citation}

Lutz, Marlon R. Jr; Zeller, Matthias; and Becker, Daniel P. Ph.D.. 2,3,8,12,13-Pentamethoxy-5H-dibenzo-

[c,n]acridin-7(6H)-one toluene solvate. Acta Crystallographica Section E: Crystalographic

Communications, E63, : o3857-o3858, 2007. Retrieved from Loyola eCommons, Chemistry: Faculty

Publications and Other Works, http://dx.doi.org/10.1107/S1600536807040548

This Article is brought to you for free and open access by the Faculty Publications and Other Works by Department at Loyola eCommons. It has been accepted for inclusion in Chemistry: Faculty Publications and Other Works by an authorized administrator of Loyola eCommons. For more information, please contact ecommons@luc.edu. cc) (i) $\Theta$

This work is licensed under a Creative Commons Attribution-Noncommercial-No Derivative Works 3.0 License. (c) International Union of Crystallography, 2007. 
Submitted to Acta Cryst. E

\title{
2,3,8,12,13-Pentamethoxy-5H-dibenzo[c,n]acridin-7(6H)-one
}

\author{
Marlon R Lutz Jr. ${ }^{\text {, }}$, Matthias Zeller ${ }^{b}$ and Daniel P. Becker ${ }^{a}$ \\ ${ }^{\mathbf{a} D e p a r t m e n t}$ of Chemistry, Loyola University, 6525 North Sheridan Road, Chicago, IL 60626, USA, and ${ }^{\mathbf{b}}$ Department of Chemistry, \\ Youngstown State University, One University Plaza, Youngstown, $\mathrm{OH} 44555$, USA \\ Correspondence email: dbecke3@luc.edu
}

\begin{abstract}
The title compound, $\mathrm{C}_{26} \mathrm{H}_{25} \mathrm{NO}_{6}$, formed by an unexpected tandem reaction of Beckmann rearrangement, electrophilic aromatic addition and subsequent demethylation, was crystallized as its toluene solvate. The crystal under investigation was found to be non-merohedrally twinned by a rotation around the reciprocal axis [ $\left[\begin{array}{ll}1 & 0\end{array}\right]$, the twin ratio refined to 0.688 (2) to 0.312 (2). The compound shows an unusual helical arrangement of three six-membered rings that are all connected at the central carbon atom C6. The helix effectively performs one full turn around C6, and the thread pitch, as defined by the distance of the terminal atoms C2 and C20 of the helix, is 4.98 (3) $\AA$. The angles around C6 are between 104.7 (2) and $115.2(2)^{\circ}$. The middle ring, a cyclohexa-2,4-dienimine with $\mathrm{C} 6$ being the only saturated atom in the ring, is nearly planar with an r.m.s. deviation from the mean plane of only $0.035 \AA$. The other two rings have conformations best described as between envelope and screw-boat with puckering amplitudes of 0.527 (2) and 0.544 (2) $\AA$, respectively.
\end{abstract}

\section{Related literature}

For the use of crown-shaped [1.1.1]orthocyclophane cyclotriveratrylene (CTV, hexamethoxy tribenzocyclononene) as a scaffold in supramolecular chemistry see Collet, 1987. For crown and saddle conformers of CTV oxime see Lutz et al., 2007.

\section{Computing details}

Data collection: SMART (Bruker, 1997-2002); cell refinement: Cell_Now (Sheldrick, 2004); SAINT+ (Bruker, 2003); data reduction: SAINT+; program(s) used to solve structure: SHELXTL (Bruker, 2000-2003); program(s) used to refine structure: SHELXTL; molecular graphics: SHELXTL; software used to prepare material for publication: SHELXTL.

\section{(2,3,8,12,13-Pentamethoxy-5H-dibenzoc,nacridin-76H-one)}

Crystal data

$\mathrm{C}_{26} \mathrm{H}_{25} \mathrm{NO}_{6} \cdot \mathrm{C}_{7} \mathrm{H}_{8}$

$M_{r}=539.60$

$V=2757.1(12) \AA^{3}$

Monoclinic, $P 2{ }_{1} / c$

$Z=4$

Mo $K \alpha$ 


\section{Acta E preprint}

$$
\begin{aligned}
& a=14.952(4) \AA \\
& b=7.1736(18) \AA \\
& c=25.787(6) \AA \\
& \beta=94.571(7)^{\circ}
\end{aligned}
$$

\section{Data collection}

\section{Bruker AXS SMART APEX CCD} diffractometer

Absorption correction: multi-scan TWINABS (Bruker, 2003)

$T_{\min }=0.773, T_{\max }=1.000$

40178 measured reflections

$$
\begin{aligned}
& \mu=0.09 \mathrm{~mm}^{-1} \\
& T=100(2) \mathrm{K} \\
& 0.60 \times 0.19 \times 0.09 \mathrm{~mm}
\end{aligned}
$$

10641 independent reflections

7887 reflections with $I>2 \sigma(I)$

$R_{\text {int }}=$ not defined due to twin pairing errors, HerbstIrmer, 2006

\section{Refinement}

$R\left[F^{2}>2 \sigma\left(F^{2}\right)\right]=0.076$ 368 parameters

$w R\left(F^{2}\right)=0.199$ $\mathrm{H}$-atom parameters constrained

$S=1.08$

$\Delta \rho_{\max }=0.67$ e $\AA^{-3}$

10641 reflections

Table 1. Hydrogen bonding and $C-H \cdots \pi$ interactions

$\begin{array}{lllll}\mathrm{D}-\mathrm{H} \cdots \mathrm{A} & \mathrm{D}-\mathrm{H} & \mathrm{H} \cdots \mathrm{A} & \mathrm{D} \cdots \mathrm{A} & \mathrm{D}-\mathrm{H} \cdots \mathrm{A} \\ \mathrm{C} 23-\mathrm{H} 23 \mathrm{~B} \cdots \mathrm{O} 4^{\mathrm{i}} & 0.98 & 2.41 & 3.354(3) & 160.4 \\ \mathrm{C} 26-\mathrm{H} 26 \mathrm{~B} \cdots \mathrm{O} 2^{\mathrm{ii}} & 0.98 & 2.59 & 3.210(3) & 120.9 \\ \mathrm{C} 26-\mathrm{H} 26 \mathrm{C} \cdots \mathrm{O} 6^{\mathrm{iii}} & 0.98 & 2.58 & 3.526(3) & 162.2 \\ \mathrm{C} 12-\mathrm{H} 12 \cdots C g(5)^{\mathrm{iV}} & 0.95 & 3.3199 & 3.780(2) & 112.02 \\ \mathrm{C} 22-\mathrm{H} 22 \mathrm{~A} \cdots \mathrm{Cg}(5)^{\mathrm{iii}} & 0.98 & 2.9687 & 3.557(3) & 119.73 \\ \mathrm{C} 23-\mathrm{H} 23 \mathrm{C} \cdots \mathrm{Cg}(4)^{\mathrm{v}} & 0.98 & 2.9869 & 3.902(3) & 155.82 \\ \mathrm{C} 24-\mathrm{H} 24 \mathrm{~A} \cdots \mathrm{Cg}(6)^{\mathrm{vi}} & 0.98 & 2.6627 & 3.447(3) & 137.12 \\ \mathrm{C} 25-\mathrm{H} 25 \mathrm{C} \cdots C g(6) & 0.98 & 2.6327 & 3.487(3) & 145.87\end{array}$

$C g(4)$ denotes the centroid of ring C8/9/10/11/12/13, $C g(5)$ that of C15/16/17/18/19/20, and $C g(6)$ that of $C 27 / 28 / 29 / 30 / 31 / 32)$. Symmetry codes: (i) $-x,-y,-z+1$; (ii) $-x+1, y+1 / 2,-z+1 / 2$; (iii) $-x+1,-y+2,-z+1$; (iv) $x, y-1, z$; (v) $-x,-y+1,-z+1$; (vi) $-x, y$ $-1 / 2,-z+1 / 2$.

\section{Acknowledgements}

The diffractometer was funded by NSF grant 0087210, by Ohio Board of Regents grant CAP-491, and by Youngstown State University.

\section{References}

Boeyens, J. C. A. (1978). J. Cryst. Mol. Struct. 8, 317-320.

Bruker (1997-2002). SMART for WNT/2000 5.630. Bruker AXS Inc, Madison (WI), USA.

Bruker (2003). SAINT+ 6.45 (includes Twinabs as an implemented sub-program). Bruker AXS Inc, Madison (WI), USA. 
Bruker (2000). SHELXTL 6.10. Bruker AXS Inc, Madison (WI), USA.

Collet, A. (1987). Tetrahedron, 43, 5725-5759.

Cremer, D. \& Pople, J. A. (1975). J. Amer. Chem. Soc. 97, 1354-1358.

Herbst-Irmer, R. (2006). Private Communication at the $62^{\text {th }}$ Conference of the Pittsburgh Diffraction Society, Pittsburgh (PA), USA.

Herbstein, F. H. (2000). Acta Cryst. B56, 547-557.

Lutz, M. R. Jr, French, D. C., Rehage, P. \& Becker, D. P. (2007). Tetrahedron Lett. 48(36), 6368-6371.

Sheldrick, G. M. (2004). CELL_NOW. Indexing Program for Twinned Samples. University of Göttingen, Germany. 


\title{
2,3,8,12,13-Pentamethoxy-5H-dibenzo[c,n]acridin-7 (6H)-one
}

\author{
Marlon R Lutz Jr. ${ }^{a}$, Matthias Zeller ${ }^{b}$ and Daniel P. Becker ${ }^{a}$
}

\section{Comment}

The crown-shaped [1.1.1]orthocyclophane cyclotriveratrylene (CTV, hexamethoxy tribenzocyclononene) has been employed extensively as a scaffold in supramolecular chemistry (Collet, 1987). We are interested in new apex-modified derivatives of CTV and recently reported the isolation of the crown and saddle conformers of CTV oxime (Lutz et al., 2007). In the course of studying the Beckmann rearrangement of this molecule we observed the unexpected formation of the title compound I, resulting from a Beckmann rearrangement followed by an intramolecular electrophilic aromatic addition and subsequent demethylation (Figure 1). Studies of the reaction conditions and mechanism will be discussed in detail in a separate publication.

Compound I, $\mathrm{C}_{26} \mathrm{H}_{25} \mathrm{NO}_{6}$, was crystallized from methylene chloride/toluene as its toluene solvate (Figure 2). The red needle-like crystals were heavily intergrown, and the crystal that was finally selected for single-crystal data collection was found to be non-merohedrally twinned with two twin components in a ratio of 0.688 (2) to 0.312 (2) (See experimental refinement section for details of unit cell determination, data workup, refinement, and type of twinning).

The compound shows an unusual helical arrangement of three six-membered rings that are all connected at the central carbon atom C6. The helix effectively performs one full turn around C6, and the thread pitch, as defined by the distance of the terminal atoms $\mathrm{C} 2$ and $\mathrm{C} 20$ of the helix, is 4.98 (3) $\AA$. The angles around C6 are between 104.7 (2) and $115.2(2)^{\circ}$. The middle ring, a cyclohexa-2,4-dienimine with $\mathrm{C} 6$ being the only saturated atom in the ring, is nearly planar with an r.m.s. deviation from the mean plane of only $0.035 \AA$. The other two rings have conformations best described as between envelope and screw-boat (Boeyens, 1978) with puckering amplitudes of 0.527 (2) and 0.544 (2) $\AA$, respectively (Cremer \& Pople, 1975).

The packing of (I), illustrated in Figure 3, seems to be dominated by a combination of simple dispersion forces, and weak interactions of the methoxy methyl hydrogen bonds with both neigboring oxygen atoms and aromatic rings. All methoxy groups are involved in at least one $\mathrm{C}-\mathrm{H} \cdots \mathrm{O}$ hydrogen bond or $\mathrm{C}-\mathrm{H} \cdots \pi$ contact, and all aromatic rings act as an acceptor to one or two methoxy $\mathrm{CH}_{3}$ groups (see Table 1 for a list and numerical values).

\section{Experimental}

To a solution of the crown conformer of CTV oxime (10,15-dihydro-2,3,7,8,12,13-hexamethoxy-5Htribenzo[a,d,g]cyclononen-5-oxime, $200 \mathrm{mg}, 0.417 \mathrm{mmol}$ ) in $4 \mathrm{ml}$ diethyl ether and $1 \mathrm{ml}$ of dichloromethane at $273 \mathrm{~K}$ was added thionyl chloride $(1.88 \mathrm{~g}, 15.8 \mathrm{mmol})$ dropwise over 1 minute. The reaction mixture was stirred for 5 minutes at 273 $\mathrm{K}$, then poured over ice and extracted with methylene chloride. The organic layer was washed successively with saturated aqueous sodium bicarbonate, water and brine, and then dried over sodium sulfate. Concentration gave a residue which was chromatographed on silica gel eluting with ethyl acetate/methylene chloride (30/70) to afford $0.028 \mathrm{~g}$ of a solid which was recrystallized from toluene/dichloromethane to give $0.023 \mathrm{~g} \mathrm{(23 \% )}$ of the product as red-bronze crystals. 


\section{supplementary materials}

\section{Refinement}

The crystal under investigation was found to be non-merohedrally twinned. The orientation matrices for the two components were identified using the program Cell_Now (Sheldrick, 2004), and the two components were integrated using Saint+ (Bruker, 2003), resulting in a total of 40178 reflections. 12959 reflections (5474 unique ones) involved component 1 only (mean I/sigma $=5.5), 12616$ reflections (5374 unique ones) involved component 2 only (mean I/sigma $=3.7$ ), and 14603 reflections (7998 unique ones) involved both components (mean I/sigma $=6.4$ ). The exact twin matrix identified by the integration program was found to be $0.99876-0.00396-0.00012,0.002120 .998890 .00875,-0.00309-0.111630 .99795$, which is for this structure equivalent to a $180^{\circ}$ rotation around the reciprocal axis $\left[\begin{array}{lll}1 & 0 & 0\end{array}\right]$.

The data were corrected for absorption using Twinabs (Bruker, 2003), and the structure was solved using direct methods with only the non-overlapping reflections of component 1 . The structure was refined using the hklf 5 routine with all reflections of component 1 (including the overlapping ones) below a $d$-spacing threshold of $3 / 4$, resulting in a BASF value of 0.312 (2). Due to "twin pairing errors" in Saint+ (equivalent reflections being counted as overlapping for one reflection, but as not overlapping for an eqivalent one) no accurate $R_{\text {int }}$ value can be given. This also results in an incomplete merging of equivalent reflections in Twinabs, thus resulting in too many independent reflections. (Herbst-Irmer, 2006)

Hydrogen atoms were added in calculated positions with $\mathrm{C}-\mathrm{H}$ distances of $0.95,0.99$ and $0.98 \AA$ for aromatic, methylene and methyl $\mathrm{H}$ atoms, respectively, and were refined with $U_{\text {iso }}(\mathrm{H})=x U_{\text {eq }}(\mathrm{C})\left(x=1.2\right.$ for $\mathrm{C}-\mathrm{H}$ and $\mathrm{CH}_{2}, 1.5$ for $\left.\mathrm{CH}_{3}\right)$.

The s.u. values of the cell parameters are taken from the software recognizing that the values are unreasonably small (Herbstein, 2000).

\section{Figures}

Figure 1. Synthesis of the title compound.

Figure 2. ORTEP representation of the title compound with the atomic numbering scheme. Thermal displacement parameters are at the $50 \%$ probabilty level.

Figure 3. Packing diagram of (I) with 50\% probability thermal ellipsoids. View along the $a$ axis.

\section{(2,3,8,12,13-Pentamethoxy-5H-dibenzoc,nacridin-76H-one)}

$\begin{array}{ll}\text { Crystal data } & \\ \mathrm{C}_{26} \mathrm{H}_{25} \mathrm{NO}_{6} \cdot \mathrm{C}_{7} \mathrm{H}_{8} & F_{000}=1144 \\ M_{r}=539.60 & D_{\mathrm{x}}=1.300 \mathrm{Mg} \mathrm{m}{ }^{-3} \\ & \text { Mo Ka radiation } \\ \text { Monoclinic, } P 2{ }_{1} / c & \lambda=0.71073 \AA \\ a=14.952(4) \AA & \text { Cell parameters from } 6322 \text { reflections } \\ b=7.1736(18) \AA & \theta=3.0-30.5^{\circ} \\ c=25.787(6) \AA & \mu=0.09 \mathrm{~mm}^{-1} \\ \beta=94.571(7)^{\circ} & T=100(2) \mathrm{K} \\ V=2757.1(12) \AA^{3} & \text { Rod, red } \\ Z=4 & 0.60 \times 0.19 \times 0.09 \mathrm{~mm}\end{array}$




\section{Data collection}

\section{Bruker AXS SMART APEX CCD} diffractometer

Radiation source: fine-focus sealed tube

Monochromator: graphite

$T=100(2) \mathrm{K}$

$\omega$ scans

Absorption correction: multi-scan

TWINABS (Bruker, 2003)

$T_{\min }=0.773, T_{\max }=1.000$

40178 measured reflections

\section{Refinement}

Refinement on $F^{2}$

Least-squares matrix: full

$R\left[F^{2}>2 \sigma\left(F^{2}\right)\right]=0.076$

$w R\left(F^{2}\right)=0.199$

$S=1.08$

10641 reflections

368 parameters

Primary atom site location: structure-invariant direct methods
10641 independent reflections

7887 reflections with $I>2 \sigma(I)$

$R_{\text {int }}=$ not defined due to twin pairing errors, Herbst-

Irmer, 2006

$\theta_{\max }=28.3^{\circ}$

$\theta_{\min }=1.4^{\circ}$

$h=-19 \rightarrow 19$

$k=0 \rightarrow 9$

$l=0 \rightarrow 34$
Secondary atom site location: difference Fourier map

Hydrogen site location: inferred from neighbouring sites

$\mathrm{H}$-atom parameters constrained

$$
w=1 /\left[\sigma^{2}\left(F_{\mathrm{o}}^{2}\right)+(0.0913 P)^{2}+1.3281 P\right]
$$

where $P=\left(F_{\mathrm{o}}{ }^{2}+2 F_{\mathrm{c}}{ }^{2}\right) / 3$

$(\Delta / \sigma)_{\max }<0.001$

$\Delta \rho_{\max }=0.67$ e $\AA^{-3}$

$\Delta \rho_{\min }=-0.25$ e $\AA^{-3}$

Extinction correction: none

\section{Special details}

Experimental. The crystal under investigation was found to be non-merohedrally twinned. The orientation matrices for the two components were identified using the program Cell_Now, and the two components were integrated using Saint, resulting in a total of 40178 reflections. 12959 reflections (5474 unique ones) involved component 1 only (mean I/sigma $=5.5)$, 12616 reflections $(5374$ unique ones) involved component 2 only (mean I/sigma $=3.7)$, and 14603 reflections (7998 unique ones) involved both components $($ mean $\mathrm{I} /$ sigma $=6.4)$. The exact twin matrix identified by the integration program was found to be $0.99876-0.00396-0.00012$, $0.002120 .998890 .00875,-0.00309-0.111630 .99795$.

The data were corrected for absorption using twinabs, and the structure was solved using direct methods with only the non-overlapping reflections of component 1 . The structure was refined using the hklf 5 routine with all reflections of component 1 (including the overlapping ones) below a $d$-spacing threshold of $3 / 4$, resulting in a BASF value of 0.312 (2).

Geometry. All e.s.d.'s (except the e.s.d. in the dihedral angle between two 1.s. planes) are estimated using the full covariance matrix. The cell e.s.d.'s are taken into account individually in the estimation of e.s.d.'s in distances, angles and torsion angles; correlations between e.s.d.'s in cell parameters are only used when they are defined by crystal symmetry. An approximate (isotropic) treatment of cell e.s.d.'s is used for estimating e.s.d.'s involving l.s. planes.

Refinement. Refinement of $\mathrm{F}^{2}$ against ALL reflections. The weighted $R$-factor $w R$ and goodness of fit $\mathrm{S}$ are based on $\mathrm{F}^{2}$, conventional $R$-factors $R$ are based on $\mathrm{F}$, with $\mathrm{F}$ set to zero for negative $\mathrm{F}^{2}$. The threshold expression of $\mathrm{F}^{2}>2$ sigma( $\left.\mathrm{F}^{2}\right)$ is used only for calculat- 
ing $R$-factors (gt) etc. and is not relevant to the choice of reflections for refinement. $R$-factors based on $\mathrm{F}^{2}$ are statistically about twice as large as those based on F, and R- factors based on ALL data will be even larger.

Fractional atomic coordinates and isotropic or equivalent isotropic displacement parameters $\left(\AA^{2}\right)$

\begin{tabular}{|c|c|c|c|c|}
\hline & $x$ & $y$ & $z$ & $U_{\text {iso }} * / U_{\text {eq }}$ \\
\hline $\mathrm{C} 1$ & $0.36910(14)$ & $0.6758(3)$ & $0.48902(8)$ & $0.0190(4)$ \\
\hline $\mathrm{H} 1 \mathrm{~A}$ & 0.3447 & 0.5551 & 0.4757 & $0.023^{*}$ \\
\hline H1B & 0.4282 & 0.6947 & 0.4751 & $0.023^{*}$ \\
\hline $\mathrm{C} 2$ & $0.38116(14)$ & $0.6689(3)$ & $0.54776(8)$ & $0.0202(4)$ \\
\hline $\mathrm{C} 3$ & 0.38050 & 0.8509 (3) & $0.57581(8)$ & 0.0193 \\
\hline $\mathrm{C} 4$ & $0.36991(14)$ & $1.0121(3)$ & $0.54819(8)$ & $0.0192(4)$ \\
\hline $\mathrm{H} 4$ & 0.3771 & 1.1280 & 0.5659 & $0.023^{*}$ \\
\hline $\mathrm{C} 5$ & $0.34790(13)$ & $1.0112(3)$ & $0.49225(7)$ & $0.0164(4)$ \\
\hline C6 & $0.30468(14)$ & $0.8353(3)$ & $0.46952(7)$ & $0.0161(4)$ \\
\hline $\mathrm{C} 7$ & $0.21107(14)$ & $0.7989(3)$ & $0.48916(8)$ & 0.0175 \\
\hline H7A & 0.2157 & 0.7918 & 0.5276 & $0.021 *$ \\
\hline H7B & 0.1696 & 0.9016 & 0.4782 & $0.021 *$ \\
\hline $\mathrm{C} 8$ & $0.17650(14)$ & $0.6175(3)$ & $0.46620(8)$ & $0.0174(4)$ \\
\hline C9 & $0.12927(14)$ & $0.4885(3)$ & $0.49419(8)$ & $0.0193(4)$ \\
\hline H9 & 0.1164 & 0.5165 & 0.5288 & $0.023^{*}$ \\
\hline $\mathrm{C} 10$ & $0.10097(14)$ & $0.3203(3)$ & $0.47201(8)$ & $0.0191(4)$ \\
\hline $\mathrm{C} 11$ & $0.11910(14)$ & $0.2807(3)$ & $0.42046(8)$ & $0.0183(4)$ \\
\hline $\mathrm{C} 12$ & $0.16534(13)$ & $0.4081(3)$ & $0.39220(7)$ & $0.0169(4)$ \\
\hline H12 & 0.1769 & 0.3814 & 0.3573 & $0.020^{*}$ \\
\hline C13 & $0.19494(13)$ & $0.5765(3)$ & $0.41539(7)$ & $0.0165(4)$ \\
\hline $\mathrm{C} 14$ & $0.28966(13)$ & $0.8315(3)$ & 0.40988 (7) & $0.0156(4)$ \\
\hline $\mathrm{C} 15$ & $0.32871(13)$ & $0.9817(3)$ & $0.37944(7)$ & 0.0159 (4) \\
\hline $\mathrm{C} 16$ & $0.32393(13)$ & $0.9731(3)$ & $0.32478(7)$ & $0.0159(4)$ \\
\hline H16 & 0.2972 & 0.8679 & 0.3074 & $0.019 *$ \\
\hline $\mathrm{C} 17$ & $0.35782(13)$ & $1.1168(3)$ & $0.29594(8)$ & $0.0160(4)$ \\
\hline $\mathrm{C} 18$ & $0.39549(13)$ & $1.2758(3)$ & $0.32219(8)$ & $0.0167(4)$ \\
\hline C19 & $0.39815(13)$ & $1.2865(3)$ & $0.37567(8)$ & $0.0172(4)$ \\
\hline H19 & 0.4221 & 1.3948 & 0.3929 & $0.021 *$ \\
\hline $\mathrm{C} 20$ & $0.36590(13)$ & $1.1392(3)$ & $0.40523(8)$ & $0.0156(4)$ \\
\hline $\mathrm{C} 21$ & $0.37340(14)$ & $1.1502(3)$ & $0.46147(8)$ & $0.0180(4)$ \\
\hline $\mathrm{H} 21$ & 0.3974 & 1.2606 & 0.4774 & $0.022 *$ \\
\hline $\mathrm{C} 22$ & $0.41041(17)$ & $1.0041(3)$ & $0.65651(8)$ & $0.0256(5)$ \\
\hline $\mathrm{H} 22 \mathrm{~A}$ & 0.4633 & 1.0682 & 0.6452 & $0.038^{*}$ \\
\hline $\mathrm{H} 22 \mathrm{~B}$ & 0.4197 & 0.9764 & 0.6938 & $0.038^{*}$ \\
\hline $\mathrm{H} 22 \mathrm{C}$ & 0.3576 & 1.0843 & 0.6501 & $0.038^{*}$ \\
\hline $\mathrm{C} 23$ & $0.05158(16)$ & $0.2025(3)$ & $0.55146(8)$ & $0.0254(5)$ \\
\hline $\mathrm{H} 23 \mathrm{~A}$ & 0.1125 & 0.2149 & 0.5681 & $0.038^{*}$ \\
\hline $\mathrm{H} 23 \mathrm{~B}$ & 0.0231 & 0.0913 & 0.5649 & $0.038^{*}$ \\
\hline $\mathrm{H} 23 \mathrm{C}$ & 0.0164 & 0.3131 & 0.5590 & $0.038^{*}$ \\
\hline $\mathrm{C} 24$ & $0.09328(15)$ & $0.0706(3)$ & $0.34878(8)$ & $0.0221(5)$ \\
\hline $\mathrm{H} 24 \mathrm{~A}$ & 0.0601 & 0.1665 & 0.3281 & $0.033^{*}$ \\
\hline H24B & 0.0664 & -0.0517 & 0.3408 & $0.033^{*}$ \\
\hline
\end{tabular}




\begin{tabular}{|c|c|c|c|c|}
\hline $\mathrm{H} 24 \mathrm{C}$ & 0.1560 & 0.0701 & 0.3403 & $0.033^{*}$ \\
\hline $\mathrm{C} 25$ & $0.32396(16)$ & $0.9605(3)$ & $0.21512(8)$ & $0.0251(5)$ \\
\hline $\mathrm{H} 25 \mathrm{~A}$ & 0.3573 & 0.8492 & 0.2274 & $0.038 *$ \\
\hline $\mathrm{H} 25 \mathrm{~B}$ & 0.3303 & 0.9779 & 0.1779 & $0.038^{*}$ \\
\hline $\mathrm{H} 25 \mathrm{C}$ & 0.2604 & 0.9451 & 0.2208 & $0.038^{*}$ \\
\hline $\mathrm{C} 26$ & $0.46204(15)$ & $1.5756(3)$ & $0.31657(8)$ & $0.0209(5)$ \\
\hline $\mathrm{H} 26 \mathrm{~A}$ & 0.4142 & 1.6355 & 0.3344 & $0.031 *$ \\
\hline $\mathrm{H} 26 \mathrm{~B}$ & 0.4837 & 1.6616 & 0.2908 & $0.031 *$ \\
\hline $\mathrm{H} 26 \mathrm{C}$ & 0.5116 & 1.5426 & 0.3421 & $0.031 *$ \\
\hline $\mathrm{C} 27$ & $0.14872(15)$ & $0.6018(3)$ & $0.21337(8)$ & $0.0239(5)$ \\
\hline $\mathrm{C} 28$ & $0.14841(16)$ & $0.6401(4)$ & $0.16066(9)$ & $0.0299(6)$ \\
\hline $\mathrm{H} 28$ & 0.1748 & 0.5535 & 0.1385 & $0.036^{*}$ \\
\hline $\mathrm{C} 29$ & $0.11040(18)$ & $0.8023(4)$ & $0.13957(9)$ & $0.0365(6)$ \\
\hline $\mathrm{H} 29$ & 0.1103 & 0.8252 & 0.1033 & $0.044 *$ \\
\hline $\mathrm{C} 30$ & $0.07272(17)$ & $0.9308(4)$ & $0.17138(10)$ & $0.0335(6)$ \\
\hline H30 & 0.0471 & 1.0426 & 0.1571 & $0.040^{*}$ \\
\hline $\mathrm{C} 31$ & $0.07257(16)$ & $0.8953(3)$ & $0.22406(9)$ & $0.0271(5)$ \\
\hline H31 & 0.0467 & 0.9829 & 0.2461 & $0.033^{*}$ \\
\hline $\mathrm{C} 32$ & $0.11011(15)$ & $0.7325(3)$ & $0.24480(8)$ & $0.0233(5)$ \\
\hline H32 & 0.1095 & 0.7094 & 0.2810 & $0.028 *$ \\
\hline C33 & 0.18935 (19) & $0.4249(4)$ & $0.23630(10)$ & $0.0384(6)$ \\
\hline H33A & 0.2126 & 0.3497 & 0.2086 & $0.058^{*}$ \\
\hline H33B & 0.1434 & 0.3541 & 0.2529 & $0.058^{*}$ \\
\hline $\mathrm{H} 33 \mathrm{C}$ & 0.2385 & 0.4561 & 0.2623 & $0.058^{*}$ \\
\hline N1 & $0.24151(11)$ & $0.7050(2)$ & $0.38542(6)$ & $0.0174(4)$ \\
\hline $\mathrm{O} 1$ & $0.39663(10)$ & $0.8337(2)$ & $0.62800(5)$ & $0.0222(3)$ \\
\hline $\mathrm{O} 2$ & $0.35886(10)$ & $1.1202(2)$ & $0.24319(5)$ & $0.0203(3)$ \\
\hline $\mathrm{O} 3$ & $0.42770(10)$ & $1.4094(2)$ & $0.29075(5)$ & $0.0210(3)$ \\
\hline $\mathrm{O} 4$ & $0.08931(10)$ & $0.1096(2)$ & $0.40264(5)$ & $0.0222(3)$ \\
\hline O5 & $0.05546(10)$ & $0.1852(2)$ & $0.49660(5)$ & $0.0239(4)$ \\
\hline O6 & $0.39527(12)$ & $0.5216(2)$ & $0.57117(6)$ & $0.0308(4)$ \\
\hline
\end{tabular}

Atomic displacement parameters $\left(\AA^{2}\right)$

$\begin{array}{lllllll} & U^{11} & U^{22} & U^{33} & U^{12} & U^{13} & U^{23} \\ \text { C1 } & 0.0221(11) & 0.0138(10) & 0.0210(10) & 0.0001(9) & 0.0014(8) & -0.0018(8) \\ \text { C2 } & 0.0192(11) & 0.0185(11) & 0.0225(11) & -0.0013(9) & -0.0014(8) & 0.0001(8) \\ \text { C3 } & 0.0202(11) & 0.0192(11) & 0.0184(10) & -0.0038(9) & 0.0018(8) & -0.0013(8) \\ \text { C4 } & 0.0223(11) & 0.0151(10) & 0.0204(10) & -0.0038(9) & 0.0031(8) & -0.0028(8) \\ \text { C5 } & 0.0171(10) & 0.0131(10) & 0.0193(10) & -0.0005(8) & 0.0027(8) & -0.0030(8) \\ \text { C6 } & 0.0193(10) & 0.0131(10) & 0.0160(9) & -0.0019(9) & 0.0018(8) & 0.0002(7) \\ \text { C7 } & 0.0206(10) & 0.0148(10) & 0.0176(10) & -0.0014(9) & 0.0043(8) & -0.0029(8) \\ \text { C8 } & 0.0168(10) & 0.0150(10) & 0.0203(10) & 0.0002(9) & 0.0017(8) & -0.0009(8) \\ \text { C9 } & 0.0238(11) & 0.0180(10) & 0.0167(10) & -0.0026(9) & 0.0065(8) & -0.0021(8) \\ \text { C10 } & 0.0201(10) & 0.0166(10) & 0.0207(10) & -0.0026(9) & 0.0027(8) & 0.0034(8) \\ \text { C11 } & 0.0195(10) & 0.0128(10) & 0.0224(10) & -0.0032(9) & -0.0002(8) & 0.0000(8) \\ \text { C12 } & 0.0187(10) & 0.0166(10) & 0.0156(9) & -0.0003(9) & 0.0023(8) & 0.0009(8) \\ \text { C13 } & 0.0163(10) & 0.0148(10) & 0.0182(10) & -0.0013(9) & -0.0009(8) & 0.0027(8)\end{array}$




$\begin{array}{lllllll}\text { C14 } & 0.0160(10) & 0.0123(10) & 0.0187(10) & 0.0019(8) & 0.0019(8) & -0.0021(8) \\ \text { C15 } & 0.0144(10) & 0.0141(10) & 0.0195(10) & -0.0004(8) & 0.0026(7) & 0.0020(8) \\ \text { C16 } & 0.0145(9) & 0.0126(10) & 0.0207(10) & -0.0009(8) & 0.0019(8) & -0.0002(8) \\ \text { C17 } & 0.0138(10) & 0.0160(10) & 0.0182(9) & 0.0013(8) & 0.0024(7) & -0.0001(8) \\ \text { C18 } & 0.0143(10) & 0.0144(10) & 0.0214(10) & -0.0003(8) & 0.0025(8) & 0.0022(8) \\ \text { C19 } & 0.0161(10) & 0.0110(9) & 0.0245(10) & -0.0013(8) & 0.0011(8) & 0.0005(8) \\ \text { C20 } & 0.0145(10) & 0.0113(9) & 0.0212(10) & -0.0003(8) & 0.0022(8) & 0.0007(7) \\ \text { C21 } & 0.0206(10) & 0.0134(10) & 0.0200(10) & -0.0023(9) & 0.0022(8) & -0.0018(8) \\ \text { C22 } & 0.0352(13) & 0.0216(11) & 0.0198(11) & -0.0029(11) & 0.0004(9) & -0.0048(9) \\ \text { C23 } & 0.0306(12) & 0.0254(12) & 0.0206(11) & -0.0101(10) & 0.0048(9) & 0.0028(9) \\ \text { C24 } & 0.0235(11) & 0.0203(11) & 0.0226(11) & -0.0030(10) & 0.0022(8) & -0.0042(8) \\ \text { C25 } & 0.0303(13) & 0.0249(12) & 0.0203(11) & -0.0060(10) & 0.0025(9) & -0.0032(9) \\ \text { C26 } & 0.0236(11) & 0.0143(10) & 0.0252(11) & -0.0039(9) & 0.0048(9) & 0.0007(8) \\ \text { C27 } & 0.0211(11) & 0.0230(12) & 0.0275(12) & -0.0040(10) & 0.0018(9) & -0.0001(9) \\ \text { C28 } & 0.0273(13) & 0.0334(14) & 0.0301(12) & -0.0003(11) & 0.0088(10) & -0.0058(10) \\ \text { C29 } & 0.0406(15) & 0.0467(16) & 0.0224(12) & -0.0016(13) & 0.0046(10) & 0.0100(11) \\ \text { C30 } & 0.0327(14) & 0.0270(13) & 0.0403(14) & 0.0006(12) & -0.0007(11) & 0.0076(11) \\ \text { C31 } & 0.0268(12) & 0.0220(12) & 0.0323(12) & 0.0001(10) & 0.0013(10) & -0.0058(10) \\ \text { C32 } & 0.0235(11) & 0.0270(12) & 0.0194(10) & -0.0029(10) & 0.0023(8) & -0.0010(9) \\ \text { C33 } & 0.0398(15) & 0.0315(14) & 0.0447(15) & 0.0056(13) & 0.0085(12) & 0.0043(12) \\ \text { N1 } & 0.0189(9) & 0.0140(9) & 0.0198(9) & -0.0025(7) & 0.0038(7) & 0.0005(7) \\ \text { O1 } & 0.0296(8) & 0.0186(8) & 0.0180(7) & -0.0058(7) & 0.0000(6) & -0.0002(6) \\ \text { O2 } & 0.0255(8) & 0.0187(8) & 0.0173(7) & -0.0054(7) & 0.0050(6) & -0.0007(6) \\ \text { O3 } & 0.0257(8) & 0.0169(8) & 0.0210(7) & -0.0071(7) & 0.0050(6) & 0.0016(6) \\ \text { O4 } & 0.0301(9) & 0.0165(8) & 0.0204(7) & -0.0082(7) & 0.0040(6) & -0.0021(6) \\ \text { O5 } & 0.0323(9) & 0.0193(8) & 0.0212(7) & -0.0089(7) & 0.0085(6) & 0.0006(6) \\ \text { O6 } & 0.0452(11) & 0.0174(8) & 0.0282(9) & -0.0005(8) & -0.0069(7) & 0.0044(7) \\ & & & & & & \end{array}$

Geometric parameters $(\AA, \circ)$

$\begin{array}{ll}\mathrm{C} 1-\mathrm{C} 2 & 1.512(3) \\ \mathrm{C} 1-\mathrm{C} 6 & 1.553(3) \\ \mathrm{C} 1-\mathrm{H} 1 \mathrm{~A} & 0.9900 \\ \mathrm{C} 1-\mathrm{H} 1 \mathrm{~B} & 0.9900 \\ \mathrm{C} 2-\mathrm{O} 6 & 1.227(2) \\ \mathrm{C} 2-\mathrm{C} 3 & 1.493(3) \\ \mathrm{C} 3-\mathrm{O} 1 & 1.354(2) \\ \mathrm{C} 3-\mathrm{C} 4 & 1.361(3) \\ \mathrm{C} 4-\mathrm{C} 5 & 1.454(3) \\ \mathrm{C} 4-\mathrm{H} 4 & 0.9500 \\ \mathrm{C} 5-\mathrm{C} 21 & 1.348(3) \\ \mathrm{C} 5-\mathrm{C} 6 & 1.515(3) \\ \mathrm{C} 6-\mathrm{C} 14 & 1.537(3) \\ \mathrm{C} 6-\mathrm{C} 7 & 1.548(3) \\ \mathrm{C} 7-\mathrm{C} 8 & 1.504(3) \\ \mathrm{C} 7-\mathrm{H} 7 \mathrm{~A} & 0.9900 \\ \mathrm{C} 7-\mathrm{H} 7 \mathrm{~B} & 0.9900 \\ \mathrm{C} 8-\mathrm{C} 13 & 1.392(3) \\ \mathrm{C} 8-\mathrm{C} 9 & 1.399(3)\end{array}$

$\begin{array}{ll}\mathrm{C} 19-\mathrm{H} 19 & 0.9500 \\ \mathrm{C} 20-\mathrm{C} 21 & 1.448(3) \\ \mathrm{C} 21-\mathrm{H} 21 & 0.9500 \\ \mathrm{C} 22-\mathrm{O} 1 & 1.433(2) \\ \mathrm{C} 22-\mathrm{H} 22 \mathrm{~A} & 0.9800 \\ \mathrm{C} 22-\mathrm{H} 22 \mathrm{~B} & 0.9800 \\ \mathrm{C} 22-\mathrm{H} 22 \mathrm{C} & 0.9800 \\ \mathrm{C} 23-\mathrm{O} 5 & 1.426(2) \\ \mathrm{C} 23-\mathrm{H} 23 \mathrm{~A} & 0.9800 \\ \mathrm{C} 23-\mathrm{H} 23 \mathrm{~B} & 0.9800 \\ \mathrm{C} 23-\mathrm{H} 23 \mathrm{C} & 0.9800 \\ \mathrm{C} 24-\mathrm{O} 4 & 1.423(2) \\ \mathrm{C} 24-\mathrm{H} 24 \mathrm{~A} & 0.9800 \\ \mathrm{C} 24-\mathrm{H} 24 \mathrm{~B} & 0.9800 \\ \mathrm{C} 24-\mathrm{H} 24 \mathrm{C} & 0.9800 \\ \mathrm{C} 25-\mathrm{O} 2 & 1.431(2) \\ \mathrm{C} 25-\mathrm{H} 25 \mathrm{~A} & 0.9800 \\ \mathrm{C} 25-\mathrm{H} 25 \mathrm{~B} & 0.9800 \\ \mathrm{C} 25-\mathrm{H} 25 \mathrm{C} & 0.9800\end{array}$




\begin{tabular}{|c|c|c|c|}
\hline $\mathrm{C} 9-\mathrm{C} 10$ & $1.387(3)$ & $\mathrm{C} 26-\mathrm{O} 3$ & $1.440(2)$ \\
\hline C9- $\mathrm{H} 9$ & 0.9500 & $\mathrm{C} 26-\mathrm{H} 26 \mathrm{~A}$ & 0.9800 \\
\hline $\mathrm{C} 10-\mathrm{O} 5$ & $1.369(2)$ & $\mathrm{C} 26-\mathrm{H} 26 \mathrm{~B}$ & 0.9800 \\
\hline $\mathrm{C} 10-\mathrm{C} 11$ & $1.407(3)$ & $\mathrm{C} 26-\mathrm{H} 26 \mathrm{C}$ & 0.9800 \\
\hline $\mathrm{C} 11-\mathrm{O} 4$ & $1.372(2)$ & $\mathrm{C} 27-\mathrm{C} 28$ & $1.386(3)$ \\
\hline $\mathrm{C} 11-\mathrm{C} 12$ & $1.387(3)$ & $\mathrm{C} 27-\mathrm{C} 32$ & $1.394(3)$ \\
\hline $\mathrm{C} 12-\mathrm{C} 13$ & $1.404(3)$ & $\mathrm{C} 27-\mathrm{C} 33$ & $1.507(3)$ \\
\hline $\mathrm{C} 12-\mathrm{H} 12$ & 0.9500 & $\mathrm{C} 28-\mathrm{C} 29$ & $1.387(3)$ \\
\hline $\mathrm{C} 13-\mathrm{N} 1$ & $1.420(2)$ & $\mathrm{C} 28-\mathrm{H} 28$ & 0.9500 \\
\hline $\mathrm{C} 14-\mathrm{N} 1$ & $1.291(3)$ & $\mathrm{C} 29-\mathrm{C} 30$ & $1.383(4)$ \\
\hline $\mathrm{C} 14-\mathrm{C} 15$ & $1.480(3)$ & $\mathrm{C} 29-\mathrm{H} 29$ & 0.9500 \\
\hline $\mathrm{C} 15-\mathrm{C} 20$ & $1.403(3)$ & $\mathrm{C} 30-\mathrm{C} 31$ & $1.382(3)$ \\
\hline $\mathrm{C} 15-\mathrm{C} 16$ & $1.407(3)$ & $\mathrm{C} 30-\mathrm{H} 30$ & 0.9500 \\
\hline $\mathrm{C} 16-\mathrm{C} 17$ & $1.390(3)$ & $\mathrm{C} 31-\mathrm{C} 32$ & $1.385(3)$ \\
\hline $\mathrm{C} 16-\mathrm{H} 16$ & 0.9500 & $\mathrm{C} 31-\mathrm{H} 31$ & 0.9500 \\
\hline $\mathrm{C} 17-\mathrm{O} 2$ & $1.362(2)$ & $\mathrm{C} 32-\mathrm{H} 32$ & 0.9500 \\
\hline $\mathrm{C} 17-\mathrm{C} 18$ & $1.420(3)$ & $\mathrm{C} 33-\mathrm{H} 33 \mathrm{~A}$ & 0.9800 \\
\hline $\mathrm{C} 18-\mathrm{O} 3$ & $1.368(2)$ & С $33-\mathrm{H} 33 \mathrm{~B}$ & 0.9800 \\
\hline $\mathrm{C} 18-\mathrm{C} 19$ & $1.379(3)$ & $\mathrm{C} 33-\mathrm{H} 33 \mathrm{C}$ & 0.9800 \\
\hline $\mathrm{C} 19-\mathrm{C} 20$ & $1.411(3)$ & & \\
\hline $\mathrm{C} 2-\mathrm{C} 1-\mathrm{C} 6$ & $111.71(16)$ & $\mathrm{C} 15-\mathrm{C} 20-\mathrm{C} 19$ & $119.14(18)$ \\
\hline $\mathrm{C} 2-\mathrm{C} 1-\mathrm{H} 1 \mathrm{~A}$ & 109.3 & $\mathrm{C} 15-\mathrm{C} 20-\mathrm{C} 21$ & $120.99(18)$ \\
\hline $\mathrm{C} 6-\mathrm{C} 1-\mathrm{H} 1 \mathrm{~A}$ & 109.3 & $\mathrm{C} 19-\mathrm{C} 20-\mathrm{C} 21$ & $119.86(18)$ \\
\hline $\mathrm{C} 2-\mathrm{C} 1-\mathrm{H} 1 \mathrm{~B}$ & 109.3 & $\mathrm{C} 5-\mathrm{C} 21-\mathrm{C} 20$ & $123.13(19)$ \\
\hline $\mathrm{C} 6-\mathrm{C} 1-\mathrm{H} 1 \mathrm{~B}$ & 109.3 & $\mathrm{C} 5-\mathrm{C} 21-\mathrm{H} 21$ & 118.4 \\
\hline $\mathrm{H} 1 \mathrm{~A}-\mathrm{C} 1-\mathrm{H} 1 \mathrm{~B}$ & 107.9 & $\mathrm{C} 20-\mathrm{C} 21-\mathrm{H} 21$ & 118.4 \\
\hline $\mathrm{O} 6-\mathrm{C} 2-\mathrm{C} 3$ & $121.48(19)$ & $\mathrm{O} 1-\mathrm{C} 22-\mathrm{H} 22 \mathrm{~A}$ & 109.5 \\
\hline $\mathrm{O} 6-\mathrm{C} 2-\mathrm{C} 1$ & $121.57(19)$ & $\mathrm{O} 1-\mathrm{C} 22-\mathrm{H} 22 \mathrm{~B}$ & 109.5 \\
\hline $\mathrm{C} 3-\mathrm{C} 2-\mathrm{C} 1$ & $116.85(18)$ & $\mathrm{H} 22 \mathrm{~A}-\mathrm{C} 22-\mathrm{H} 22 \mathrm{~B}$ & 109.5 \\
\hline $\mathrm{O} 1-\mathrm{C} 3-\mathrm{C} 4$ & $127.01(19)$ & $\mathrm{O} 1-\mathrm{C} 22-\mathrm{H} 22 \mathrm{C}$ & 109.5 \\
\hline $\mathrm{O} 1-\mathrm{C} 3-\mathrm{C} 2$ & $113.21(18)$ & $\mathrm{H} 22 \mathrm{~A}-\mathrm{C} 22-\mathrm{H} 22 \mathrm{C}$ & 109.5 \\
\hline $\mathrm{C} 4-\mathrm{C} 3-\mathrm{C} 2$ & $119.65(18)$ & $\mathrm{H} 22 \mathrm{~B}-\mathrm{C} 22-\mathrm{H} 22 \mathrm{C}$ & 109.5 \\
\hline $\mathrm{C} 3-\mathrm{C} 4-\mathrm{C} 5$ & $121.56(19)$ & $\mathrm{O} 5-\mathrm{C} 23-\mathrm{H} 23 \mathrm{~A}$ & 109.5 \\
\hline $\mathrm{C} 3-\mathrm{C} 4-\mathrm{H} 4$ & 119.2 & $\mathrm{O} 5-\mathrm{C} 23-\mathrm{H} 23 \mathrm{~B}$ & 109.5 \\
\hline $\mathrm{C} 5-\mathrm{C} 4-\mathrm{H} 4$ & 119.2 & $\mathrm{H} 23 \mathrm{~A}-\mathrm{C} 23-\mathrm{H} 23 \mathrm{~B}$ & 109.5 \\
\hline $\mathrm{C} 21-\mathrm{C} 5-\mathrm{C} 4$ & $121.93(19)$ & $\mathrm{O} 5-\mathrm{C} 23-\mathrm{H} 23 \mathrm{C}$ & 109.5 \\
\hline $\mathrm{C} 21-\mathrm{C} 5-\mathrm{C} 6$ & $121.36(17)$ & $\mathrm{H} 23 \mathrm{~A}-\mathrm{C} 23-\mathrm{H} 23 \mathrm{C}$ & 109.5 \\
\hline $\mathrm{C} 4-\mathrm{C} 5-\mathrm{C} 6$ & $116.32(17)$ & $\mathrm{H} 23 \mathrm{~B}-\mathrm{C} 23-\mathrm{H} 23 \mathrm{C}$ & 109.5 \\
\hline $\mathrm{C} 5-\mathrm{C} 6-\mathrm{C} 14$ & $115.20(16)$ & $\mathrm{O} 4-\mathrm{C} 24-\mathrm{H} 24 \mathrm{~A}$ & 109.5 \\
\hline $\mathrm{C} 5-\mathrm{C} 6-\mathrm{C} 7$ & $112.52(16)$ & $\mathrm{O} 4-\mathrm{C} 24-\mathrm{H} 24 \mathrm{~B}$ & 109.5 \\
\hline $\mathrm{C} 14-\mathrm{C} 6-\mathrm{C} 7$ & $105.10(16)$ & $\mathrm{H} 24 \mathrm{~A}-\mathrm{C} 24-\mathrm{H} 24 \mathrm{~B}$ & 109.5 \\
\hline $\mathrm{C} 5-\mathrm{C} 6-\mathrm{C} 1$ & $104.72(16)$ & $\mathrm{O} 4-\mathrm{C} 24-\mathrm{H} 24 \mathrm{C}$ & 109.5 \\
\hline $\mathrm{C} 14-\mathrm{C} 6-\mathrm{C} 1$ & $110.39(16)$ & $\mathrm{H} 24 \mathrm{~A}-\mathrm{C} 24-\mathrm{H} 24 \mathrm{C}$ & 109.5 \\
\hline $\mathrm{C} 7-\mathrm{C} 6-\mathrm{C} 1$ & $108.88(16)$ & $\mathrm{H} 24 \mathrm{~B}-\mathrm{C} 24-\mathrm{H} 24 \mathrm{C}$ & 109.5 \\
\hline $\mathrm{C} 8-\mathrm{C} 7-\mathrm{C} 6$ & $107.96(16)$ & $\mathrm{O} 2-\mathrm{C} 25-\mathrm{H} 25 \mathrm{~A}$ & 109.5 \\
\hline $\mathrm{C} 8-\mathrm{C} 7-\mathrm{H} 7 \mathrm{~A}$ & 110.1 & $\mathrm{O} 2-\mathrm{C} 25-\mathrm{H} 25 \mathrm{~B}$ & 109.5 \\
\hline $\mathrm{C} 6-\mathrm{C} 7-\mathrm{H} 7 \mathrm{~A}$ & 110.1 & $\mathrm{H} 25 \mathrm{~A}-\mathrm{C} 25-\mathrm{H} 25 \mathrm{~B}$ & 109.5 \\
\hline $\mathrm{C} 8-\mathrm{C} 7-\mathrm{H} 7 \mathrm{~B}$ & 110.1 & $\mathrm{O} 2-\mathrm{C} 25-\mathrm{H} 25 \mathrm{C}$ & 109.5 \\
\hline $\mathrm{C} 6-\mathrm{C} 7-\mathrm{H} 7 \mathrm{~B}$ & 110.1 & $\mathrm{H} 25 \mathrm{~A}-\mathrm{C} 25-\mathrm{H} 25 \mathrm{C}$ & 109.5 \\
\hline
\end{tabular}




\begin{tabular}{|c|c|c|c|}
\hline $\mathrm{H} 7 \mathrm{~A}-\mathrm{C} 7-\mathrm{H} 7 \mathrm{~B}$ & 108.4 & $\mathrm{H} 25 \mathrm{~B}-\mathrm{C} 25-\mathrm{H} 25 \mathrm{C}$ & 109.5 \\
\hline $\mathrm{C} 13-\mathrm{C} 8-\mathrm{C} 9$ & $119.48(19)$ & $\mathrm{O} 3-\mathrm{C} 26-\mathrm{H} 26 \mathrm{~A}$ & 109.5 \\
\hline $\mathrm{C} 13-\mathrm{C} 8-\mathrm{C} 7$ & $117.74(18)$ & $\mathrm{O} 3-\mathrm{C} 26-\mathrm{H} 26 \mathrm{~B}$ & 109.5 \\
\hline $\mathrm{C} 9-\mathrm{C} 8-\mathrm{C} 7$ & $122.76(17)$ & $\mathrm{H} 26 \mathrm{~A}-\mathrm{C} 26-\mathrm{H} 26 \mathrm{~B}$ & 109.5 \\
\hline $\mathrm{C} 10-\mathrm{C} 9-\mathrm{C} 8$ & $120.85(18)$ & $\mathrm{O} 3-\mathrm{C} 26-\mathrm{H} 26 \mathrm{C}$ & 109.5 \\
\hline $\mathrm{C} 10-\mathrm{C} 9-\mathrm{H} 9$ & 119.6 & $\mathrm{H} 26 \mathrm{~A}-\mathrm{C} 26-\mathrm{H} 26 \mathrm{C}$ & 109.5 \\
\hline $\mathrm{C} 8-\mathrm{C} 9-\mathrm{H} 9$ & 119.6 & $\mathrm{H} 26 \mathrm{~B}-\mathrm{C} 26-\mathrm{H} 26 \mathrm{C}$ & 109.5 \\
\hline $\mathrm{O} 5-\mathrm{C} 10-\mathrm{C} 9$ & $124.88(18)$ & $\mathrm{C} 28-\mathrm{C} 27-\mathrm{C} 32$ & $117.9(2)$ \\
\hline $\mathrm{O} 5-\mathrm{C} 10-\mathrm{C} 11$ & $115.76(18)$ & $\mathrm{C} 28-\mathrm{C} 27-\mathrm{C} 33$ & $121.4(2)$ \\
\hline $\mathrm{C} 9-\mathrm{C} 10-\mathrm{C} 11$ & $119.36(18)$ & $\mathrm{C} 32-\mathrm{C} 27-\mathrm{C} 33$ & $120.7(2)$ \\
\hline $\mathrm{O} 4-\mathrm{C} 11-\mathrm{C} 12$ & $125.11(18)$ & $\mathrm{C} 27-\mathrm{C} 28-\mathrm{C} 29$ & $121.3(2)$ \\
\hline $\mathrm{O} 4-\mathrm{C} 11-\mathrm{C} 10$ & $114.56(17)$ & $\mathrm{C} 27-\mathrm{C} 28-\mathrm{H} 28$ & 119.3 \\
\hline $\mathrm{C} 12-\mathrm{C} 11-\mathrm{C} 10$ & $120.31(19)$ & $\mathrm{C} 29-\mathrm{C} 28-\mathrm{H} 28$ & 119.3 \\
\hline $\mathrm{C} 11-\mathrm{C} 12-\mathrm{C} 13$ & $119.77(18)$ & $\mathrm{C} 30-\mathrm{C} 29-\mathrm{C} 28$ & $120.0(2)$ \\
\hline $\mathrm{C} 11-\mathrm{C} 12-\mathrm{H} 12$ & 120.1 & $\mathrm{C} 30-\mathrm{C} 29-\mathrm{H} 29$ & 120.0 \\
\hline $\mathrm{C} 13-\mathrm{C} 12-\mathrm{H} 12$ & 120.1 & $\mathrm{C} 28-\mathrm{C} 29-\mathrm{H} 29$ & 120.0 \\
\hline $\mathrm{C} 8-\mathrm{C} 13-\mathrm{C} 12$ & $120.21(18)$ & $\mathrm{C} 31-\mathrm{C} 30-\mathrm{C} 29$ & $119.5(2)$ \\
\hline $\mathrm{C} 8-\mathrm{C} 13-\mathrm{N} 1$ & $121.20(18)$ & $\mathrm{C} 31-\mathrm{C} 30-\mathrm{H} 30$ & 120.2 \\
\hline $\mathrm{C} 12-\mathrm{C} 13-\mathrm{N} 1$ & $118.56(17)$ & $\mathrm{C} 29-\mathrm{C} 30-\mathrm{H} 30$ & 120.2 \\
\hline $\mathrm{N} 1-\mathrm{C} 14-\mathrm{C} 15$ & $118.74(17)$ & $\mathrm{C} 30-\mathrm{C} 31-\mathrm{C} 32$ & $120.2(2)$ \\
\hline $\mathrm{N} 1-\mathrm{C} 14-\mathrm{C} 6$ & $122.18(18)$ & $\mathrm{C} 30-\mathrm{C} 31-\mathrm{H} 31$ & 119.9 \\
\hline $\mathrm{C} 15-\mathrm{C} 14-\mathrm{C} 6$ & $119.04(17)$ & $\mathrm{C} 32-\mathrm{C} 31-\mathrm{H} 31$ & 119.9 \\
\hline $\mathrm{C} 20-\mathrm{C} 15-\mathrm{C} 16$ & $119.78(18)$ & $\mathrm{C} 31-\mathrm{C} 32-\mathrm{C} 27$ & $121.1(2)$ \\
\hline $\mathrm{C} 20-\mathrm{C} 15-\mathrm{C} 14$ & $119.54(17)$ & $\mathrm{C} 31-\mathrm{C} 32-\mathrm{H} 32$ & 119.5 \\
\hline $\mathrm{C} 16-\mathrm{C} 15-\mathrm{C} 14$ & $120.57(18)$ & $\mathrm{C} 27-\mathrm{C} 32-\mathrm{H} 32$ & 119.5 \\
\hline $\mathrm{C} 17-\mathrm{C} 16-\mathrm{C} 15$ & $120.79(18)$ & $\mathrm{C} 27-\mathrm{C} 33-\mathrm{H} 33 \mathrm{~A}$ & 109.5 \\
\hline $\mathrm{C} 17-\mathrm{C} 16-\mathrm{H} 16$ & 119.6 & $\mathrm{C} 27-\mathrm{C} 33-\mathrm{H} 33 \mathrm{~B}$ & 109.5 \\
\hline $\mathrm{C} 15-\mathrm{C} 16-\mathrm{H} 16$ & 119.6 & $\mathrm{H} 33 \mathrm{~A}-\mathrm{C} 33-\mathrm{H} 33 \mathrm{~B}$ & 109.5 \\
\hline $\mathrm{O} 2-\mathrm{C} 17-\mathrm{C} 16$ & $125.53(18)$ & $\mathrm{C} 27-\mathrm{C} 33-\mathrm{H} 33 \mathrm{C}$ & 109.5 \\
\hline $\mathrm{O} 2-\mathrm{C} 17-\mathrm{C} 18$ & $115.24(17)$ & $\mathrm{H} 33 \mathrm{~A}-\mathrm{C} 33-\mathrm{H} 33 \mathrm{C}$ & 109.5 \\
\hline $\mathrm{C} 16-\mathrm{C} 17-\mathrm{C} 18$ & $119.23(18)$ & $\mathrm{H} 33 \mathrm{~B}-\mathrm{C} 33-\mathrm{H} 33 \mathrm{C}$ & 109.5 \\
\hline $\mathrm{O} 3-\mathrm{C} 18-\mathrm{C} 19$ & $124.78(18)$ & $\mathrm{C} 14-\mathrm{N} 1-\mathrm{C} 13$ & $117.94(17)$ \\
\hline $\mathrm{O} 3-\mathrm{C} 18-\mathrm{C} 17$ & $115.18(17)$ & $\mathrm{C} 3-\mathrm{O} 1-\mathrm{C} 22$ & $116.05(16)$ \\
\hline $\mathrm{C} 19-\mathrm{C} 18-\mathrm{C} 17$ & $120.04(18)$ & $\mathrm{C} 17-\mathrm{O} 2-\mathrm{C} 25$ & $117.29(15)$ \\
\hline $\mathrm{C} 18-\mathrm{C} 19-\mathrm{C} 20$ & $120.98(19)$ & $\mathrm{C} 18-\mathrm{O} 3-\mathrm{C} 26$ & $115.81(15)$ \\
\hline $\mathrm{C} 18-\mathrm{C} 19-\mathrm{H} 19$ & 119.5 & $\mathrm{C} 11-\mathrm{O} 4-\mathrm{C} 24$ & $117.74(16)$ \\
\hline $\mathrm{C} 20-\mathrm{C} 19-\mathrm{H} 19$ & 119.5 & $\mathrm{C} 10-\mathrm{O} 5-\mathrm{C} 23$ & $117.31(16)$ \\
\hline $\mathrm{C} 6-\mathrm{C} 1-\mathrm{C} 2-\mathrm{O} 6$ & $148.9(2)$ & $\mathrm{N} 1-\mathrm{C} 14-\mathrm{C} 15-\mathrm{C} 20$ & $167.52(18)$ \\
\hline $\mathrm{C} 6-\mathrm{C} 1-\mathrm{C} 2-\mathrm{C} 3$ & $-34.6(3)$ & $\mathrm{C} 6-\mathrm{C} 14-\mathrm{C} 15-\mathrm{C} 20$ & $-10.2(3)$ \\
\hline $\mathrm{O} 6-\mathrm{C} 2-\mathrm{C} 3-\mathrm{O} 1$ & $-0.1(3)$ & $\mathrm{N} 1-\mathrm{C} 14-\mathrm{C} 15-\mathrm{C} 16$ & $-8.6(3)$ \\
\hline $\mathrm{C} 1-\mathrm{C} 2-\mathrm{C} 3-\mathrm{O} 1$ & $-176.55(17)$ & $\mathrm{C} 6-\mathrm{C} 14-\mathrm{C} 15-\mathrm{C} 16$ & $173.70(18)$ \\
\hline $\mathrm{O} 6-\mathrm{C} 2-\mathrm{C} 3-\mathrm{C} 4$ & $176.1(2)$ & $\mathrm{C} 20-\mathrm{C} 15-\mathrm{C} 16-\mathrm{C} 17$ & $1.7(3)$ \\
\hline $\mathrm{C} 1-\mathrm{C} 2-\mathrm{C} 3-\mathrm{C} 4$ & $-0.4(3)$ & $\mathrm{C} 14-\mathrm{C} 15-\mathrm{C} 16-\mathrm{C} 17$ & $177.83(18)$ \\
\hline $\mathrm{O} 1-\mathrm{C} 3-\mathrm{C} 4-\mathrm{C} 5$ & $-176.57(19)$ & $\mathrm{C} 15-\mathrm{C} 16-\mathrm{C} 17-\mathrm{O} 2$ & $178.08(18)$ \\
\hline $\mathrm{C} 2-\mathrm{C} 3-\mathrm{C} 4-\mathrm{C} 5$ & $7.9(3)$ & $\mathrm{C} 15-\mathrm{C} 16-\mathrm{C} 17-\mathrm{C} 18$ & $-1.6(3)$ \\
\hline $\mathrm{C} 3-\mathrm{C} 4-\mathrm{C} 5-\mathrm{C} 21$ & $-151.1(2)$ & $\mathrm{O} 2-\mathrm{C} 17-\mathrm{C} 18-\mathrm{O} 3$ & $-0.2(3)$ \\
\hline $\mathrm{C} 3-\mathrm{C} 4-\mathrm{C} 5-\mathrm{C} 6$ & $21.7(3)$ & $\mathrm{C} 16-\mathrm{C} 17-\mathrm{C} 18-\mathrm{O} 3$ & $179.55(18)$ \\
\hline $\mathrm{C} 21-\mathrm{C} 5-\mathrm{C} 6-\mathrm{C} 14$ & $-2.0(3)$ & $\mathrm{O} 2-\mathrm{C} 17-\mathrm{C} 18-\mathrm{C} 19$ & $-179.77(18)$ \\
\hline $\mathrm{C} 4-\mathrm{C} 5-\mathrm{C} 6-\mathrm{C} 14$ & $-174.90(17)$ & $\mathrm{C} 16-\mathrm{C} 17-\mathrm{C} 18-\mathrm{C} 19$ & $0.0(3)$ \\
\hline
\end{tabular}




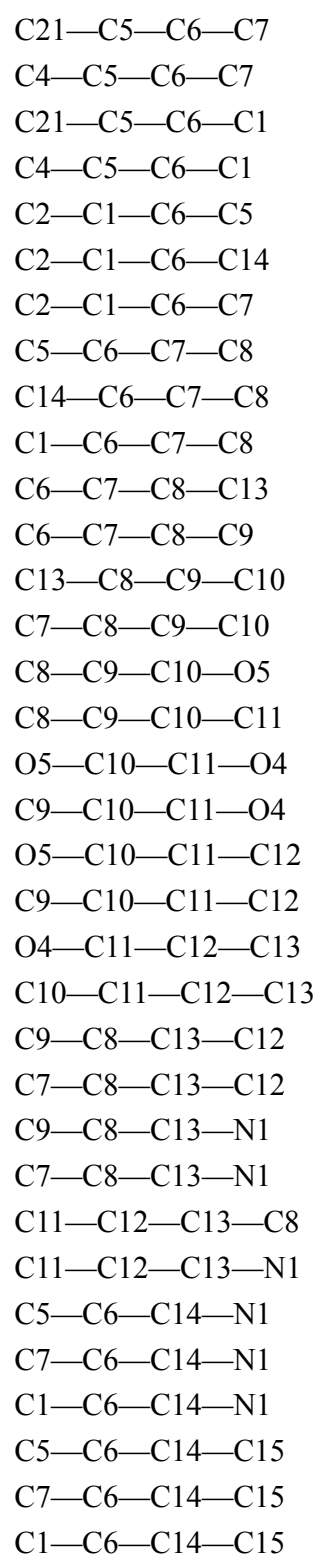

$-122.5(2)$
$64.6(2)$
$119.4(2)$
$-53.5(2)$
$58.7(2)$
$-176.78(17)$
$-61.9(2)$
$-177.45(16)$
$56.4(2)$
$-61.8(2)$
$-37.5(2)$
$140.8(2)$
$0.2(3)$
$-178.02(19)$
$179.2(2)$
$-0.9(3)$
$-0.9(3)$
$179.11(18)$
$-179.63(18)$
$0.4(3)$
$-177.92(19)$
$0.7(3)$
$0.8(3)$
$179.18(18)$
$179.03(19)$
$-2.6(3)$
$-1.3(3)$
$-179.52(18)$
$-169.12(18)$
$-44.7(2)$
$72.6(2)$
$8.5(3)$
$132.91(18)$
$-109.8(2)$

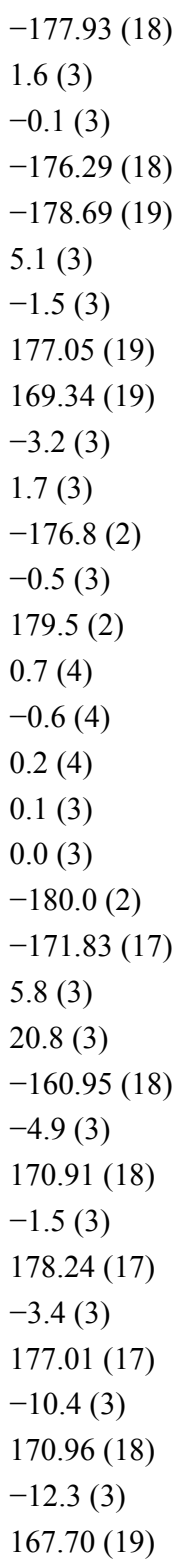




\section{2,3,8,12,13-Pentamethoxy-5H-dibenzo-[c,n]acridin-7(6H)-one toluene solvate}

Marlon R. Lutz Jr, Matthias Zeller and Daniel P. Becker*

\section{Correspondence e-mail: dbecke3@luc.edu}

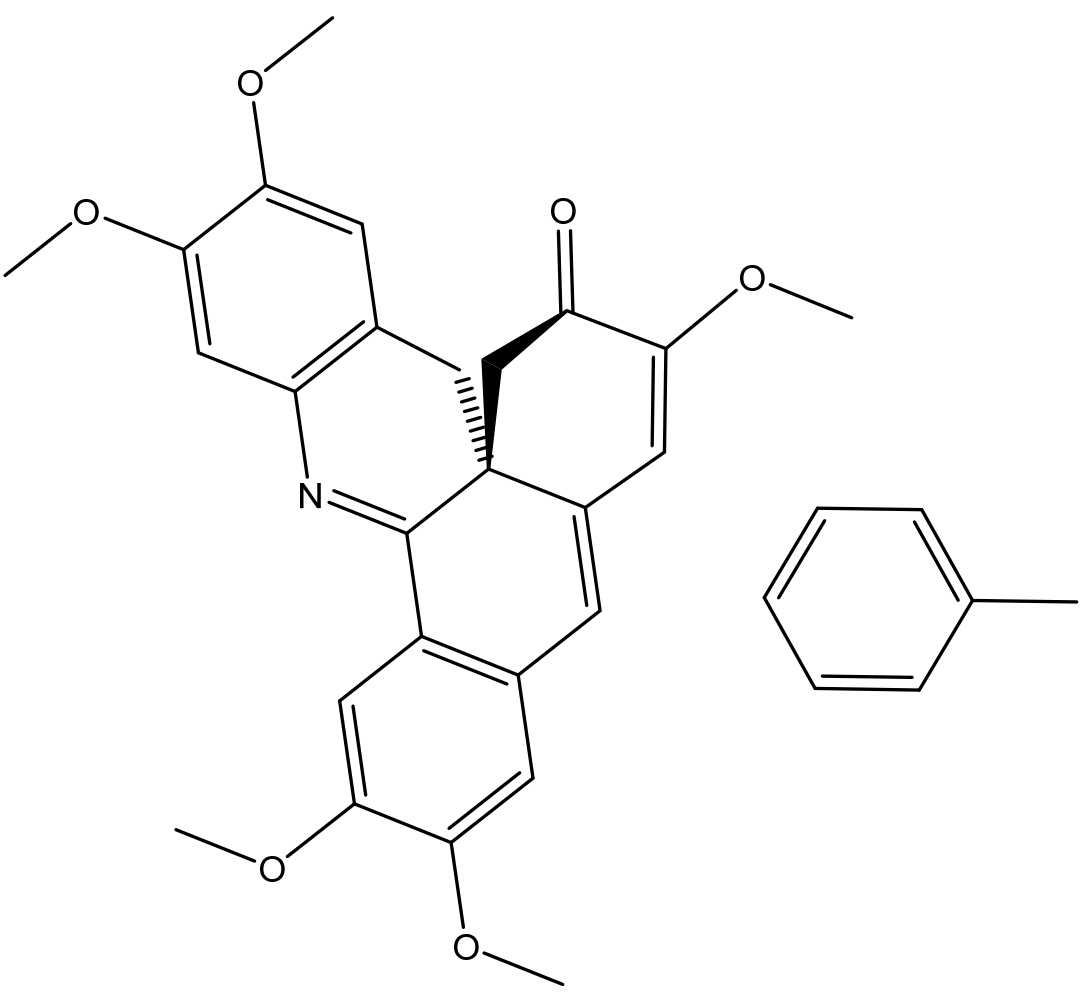




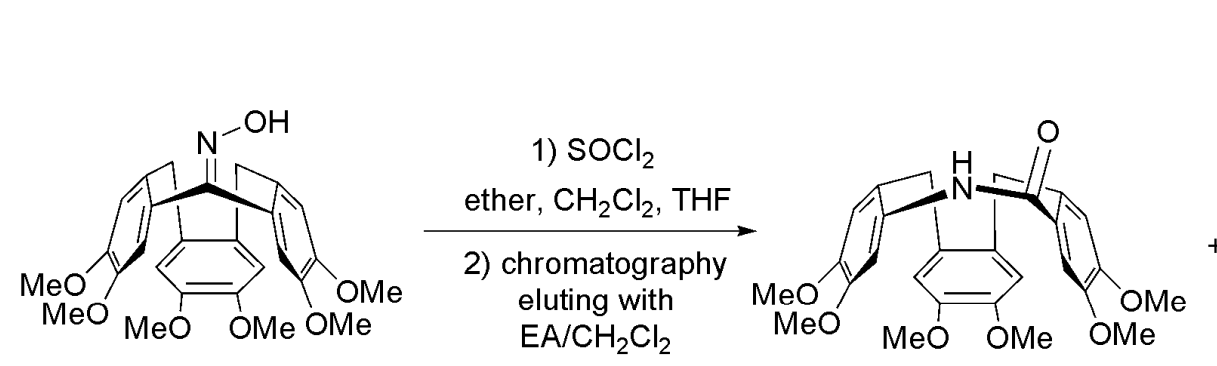

Beckmann amide

(expected product isolated separately)<smiles>COC1=CC2=Cc3cc(OC)c(OC)cc3C3=Nc4cc(OC)c(OC)cc4C3(C2)C(=O)C1=O</smiles>

Compound I

(unexpected product from cyclization reaction)

Figure 1. Synthesis of the title compound

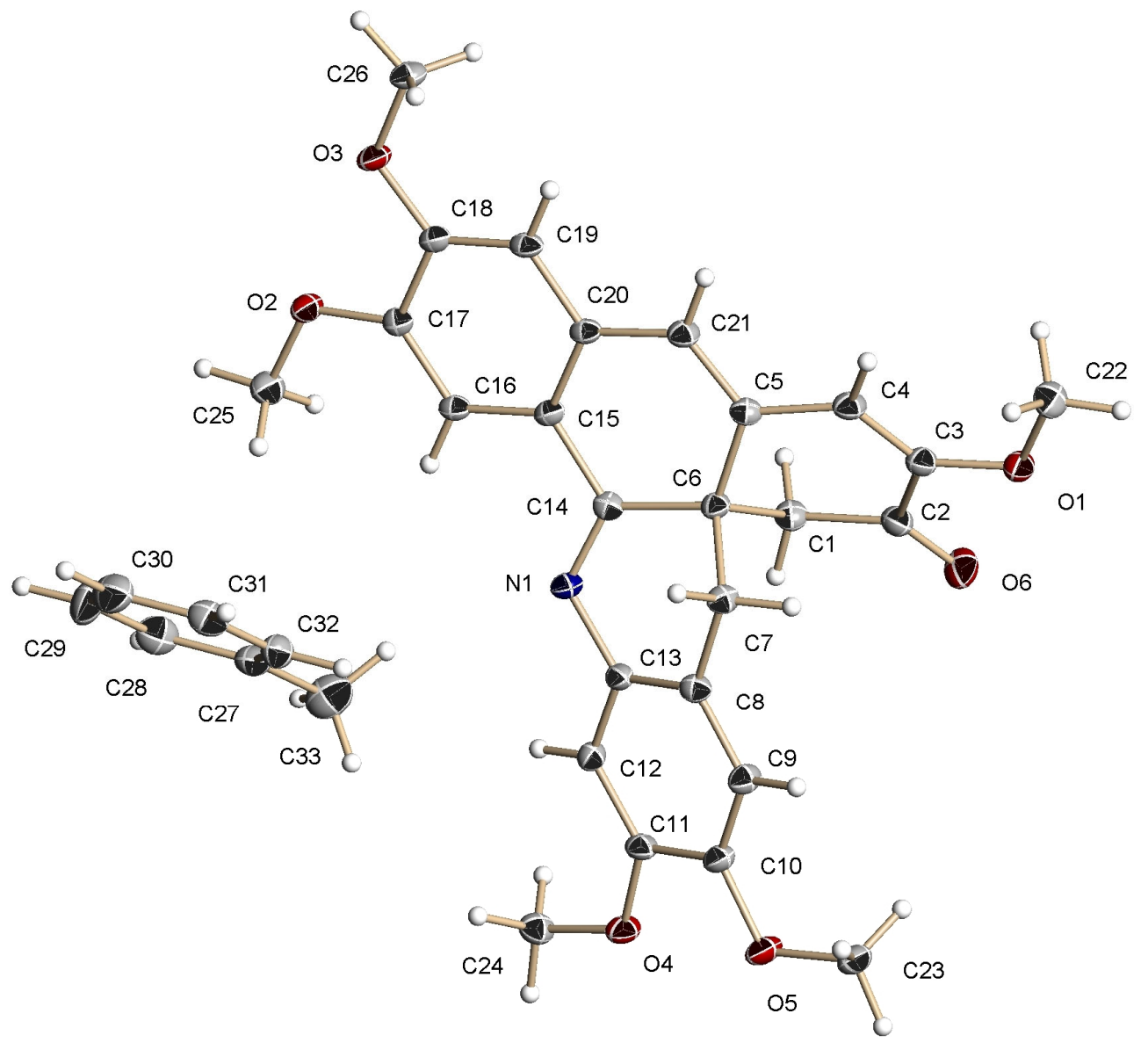

Figure 2. The molecular structure of the title compound with the atomic numbering scheme. Thermal displacement parameters are at the $50 \%$ probability level. 


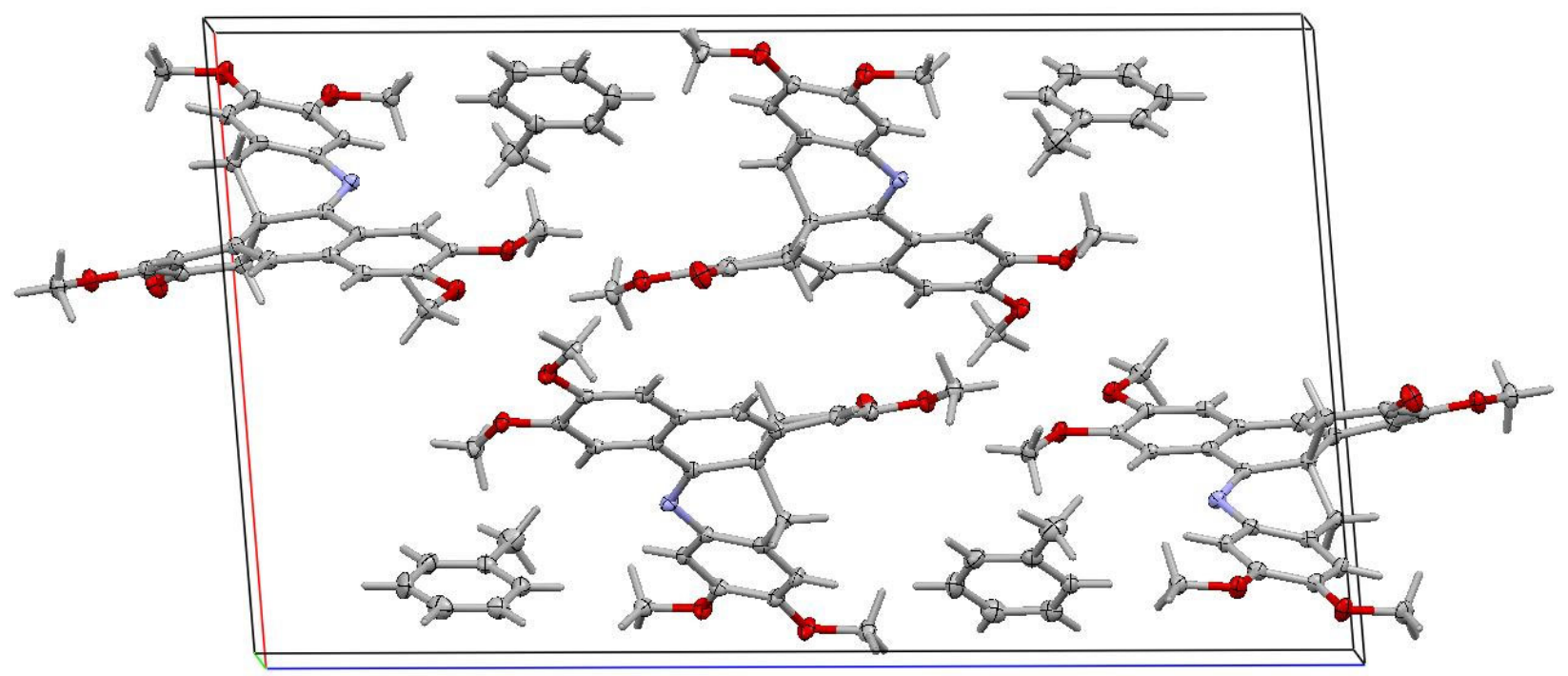

Figure 3. Partially expanded packing diagram of the title compound with $50 \%$ probability thermal ellipsoids. C-H..O hydrogen bonds are indicated by broken turquoise lines, C-H...pi contacts by dashed blue lines. 